\title{
Identification of Minuscule Inward Currents as Precursors to Membrane Electroporation-Induced Currents: Real-Time Prediction of Pore Appearance
}

\author{
Edmund Cheung So ${ }^{a, b}$ Ke-Li Tsaic Fang-Tzu Wu ${ }^{d}$ Ming-Chun Hsue King-Chuen \\ $\mathrm{Wu}^{\mathrm{f}}$ Sheng-Nan $\mathrm{Wu}^{\mathrm{e}, \mathrm{g}}$
}

\begin{abstract}
aDepartment of Anesthesia, Tainan Municipal An Nan Hospital, China Medical University, Tainan City; ${ }^{b}$ Department of Anesthesiology, China Medical University, Taichung City; 'Department of Physiology, Kaohsiung Medical University, Kaohsiung City; 'Department of Psychology, National Cheng Kung University, Tainan City; ' Department of Physiology, National Cheng Kung University Medical College, Tainan City; 'Department of Anesthesia, E-da Hospital / I-shou University, Kaohsiung City; IInstitute of Basic Medical Sciences, National Cheng Kung University Medical College, Tainan City
\end{abstract}

\section{Key Words}

Membrane electroporation - Ion current - Principal component analysis (PCA) • Palmitoyl-Lcarinitine

\begin{abstract}
Background/Aims: The objective of this study is to examine the current signals in response to large hyperpolarizations with the aid of principal component analysis (PCA) to search for or even predict current fluctuations related to membrane electroporation-induced current $\left(\mathrm{I}_{\mathrm{MEP}}\right)$. Methods: The characteristics of principal eigenvalues generated for $\mathrm{I}_{\mathrm{MEP}}$ and the current signals at $10 \mathrm{sec}$ prior to the start of initial $\mathrm{I}_{\mathrm{MEP}}\left(\mathrm{I}_{\mathrm{Pre}}\right)$ were examined. As membrane hyperpolarizations were applied at $0.1 \mathrm{~Hz}$, the appearance of $\mathrm{I}_{\text {MEP }}$ coincided with the higher principal eigenvalues extracted in PCA. Results: Subsequent addition of $\mathrm{LaCl}_{3}(100 \mu \mathrm{M})$ greatly reduced I $\mathrm{I}_{\text {MEP }}$ and associated principal eigenvalues. In real-time analysis for a single frame (i.e, $300 \mathrm{msec}$ ), in response to large hyperpolarization, multiple runs of heralded minuscule inward currents $\left(\mathrm{I}_{\min }\right)$ occurring before large rise in current amplitudes were detected. With PCA, such heralded $I_{\min }$ was noted to coincide with the extreme principal eigenvalues. The duration of $\mathrm{I}_{\min }$ together with large principal eigenvalues was influenced by different levels of membrane hyperpolarization. In GH3 cells, palmitoyl-L-carnitine (PALCAR), a long-chain acylcarnitine, effectively increased the $\mathrm{I}_{\text {MEP }}$ amplitude with an $\mathrm{EC}_{50}$ value of $2.4 \mu \mathrm{M}$. However, in PALCAR-treated cells, the $\mathrm{I}_{\text {min }}$ together with higher principal eigenvalues disappeared, while in isoflurane-treated cells, $I_{\text {min }}$ occurring before large rise of current amplitude remained intact. Similarly, the PCA analysis
\end{abstract}


from $I_{\text {Pre }}$ in RAW 264.6 macrophages showed the presence of herald $I_{\min }$ accompanied by the extreme principal eigenvalues. Conclusion: It is clear from this study that these large principal eigenvalues are representative of MEP-associated formation of electropores. Therefore, different compositions around the surface membrane of cells may alter the appearance of $I_{\text {min }}$ followed by $I_{\text {MEP }}$ emergence.

Copyright (C) 2013 S. Karger AG, Basel

\section{Introduction}

Membrane electroporation (MEP) or electro-permealization is a cellular dynamic process that consists in a significant but transient increase of the electrical conductivity and permeability of the plasma membrane by means of an externally applied electrical field. This physical phenomenon has been described in terms of structural transitions from closed to porated states through semi-permeable membranes as the transmembrane potential exceeds a critical threshold [1-3]. Such a maneuver has been employed in cancer research, gene transfection, drug delivery, and cell fusion [4, 5]. In order to efficiently electrotransfer drugs and DNAs into the cells, quantitative predictions and descriptions of mechanical, electrical, and molecular transport aspects of MEP are potentially important [3, 4]. Additionally, owing to high conductance of MEP-induced channels, even at low probability of opening, significant currents tend to flow, thereby altering the electrical behavior of cells $[1,2,6-8]$. Recent work in our laboratory has characterized the electrical properties of MEP-induced currents $\left(I_{\text {MEP }}\right)$ in different types of cells [9-12]. Besides that, despite numerous experimental and theoretical studies, many aspects of MEP-induced currents $\left(I_{\mathrm{MEP}}\right)$ remain elusive.

The detrended fluctuation analysis has been previously applied to analyze the scaling exponents in the time series of $I_{\mathrm{MEP}}$ [11]. However, its real-time computation capacity used to herald the coming of pore appearance is limited. Alternatively, principal component analysis (PCA) has been widely used in all forms of analysis ranging from neuroscience to computer graphics, because it is a simple, non-parametric method of extracting relevant information from confusing data sets $[13,14]$. With minimal additional effort, PCA is able to provide a framework for how to reduce a complex data set to a lower dimension and then to reveal the sometimes hidden, simplified structure that often underlies it. However, whether such maneuver can be applied for either the characterization of $I_{\mathrm{MEP}}$ or the prediction of pore formation remains largely unknown.

Palmitoyl-L-carnitine (PALCAR) is the most abundant long-chain acylcarnitine in ischemic tissue which may accumulate rapidly due to its ability to inhibit mitochondrial $\beta$-oxidation of fatty acid and thereby to produce toxic effects on the tissue involved [15]. Earlier studies have shown that this compound could reduce electrophoretic mobility of erythrocytes [16], alter the vectorial transport in Caco-2 cell monolayers [17], elevate intracellular $\mathrm{Ca}^{2+}$ in vascular endothelial cells and heart cells [18-20] and increase the amplitude of late $\mathrm{Na}^{+}$current [21]. However, the ability of this compound to interact with $I_{\text {MEP }}$ to influence the electrical behavior of $I_{\mathrm{MEP}}$ is incompletely understood.

Therefore, in this study, the physical properties of $I_{\text {MEP }}$ in different types of cells including pituitary tumor $\left(\mathrm{GH}_{3}\right)$ cells and RAW 264.7 macrophages were characterized with the use of patch-clamp recordings. The PCA was thereafter applied to analyze the principal eigenvalues in the time series of hyperpolarization-induced inward current through MEPinduced channels in these cells. The results showed that there exist heralded minuscule inward currents $\left(I_{\min }\right)$ immediately prior to escalating rise of current amplitude in response to membrane hyperpolarization. We also found that PALCAR was effective at stimulating $I_{\text {MEP }}$ in pituitary $\mathrm{GH}_{3}$ cells. If this approach shown here continues to prove successful, it could be used in real-time prediction of $I_{\text {MEP }}$ occurrence through early detection of MEP-associated current signals. 


\section{Materials and Methods}

\section{Drugs and Solutions}

Glibenclamide, lysophosphatidylcholine, palmitoyl-L-carnitine (PALCAR), ranolazine, riluzole and tetrodotoxin were obtained from Sigma-Aldrich (St. Louis, MO), apamin and iberiotoxin were from Alomone Labs (Jerusalem, Israel), and isoflurane (Florane ${ }^{\circledR}$ ) was from Abbott Lab. (Taipei, Taiwan). Prior to the experiment, isoflurane was mixed to graduated syringes with the appropriate bath solution. All culture media, fetal calf serum, L-glutamine, trypsin/EDTA, and penicillin-streptomycin were obtained from Invitrogen (Carlsbad, CA). Other chemicals including $\mathrm{CsCl}, \mathrm{CsOH}, \mathrm{LaCl}_{3}, \mathrm{MnCl}_{2}$ and $\mathrm{N}$-methyl-D-glucamine ${ }^{+}\left(\mathrm{NMDG}^{+}\right.$) were commercially available and of reagent grade. Deionized water used throughout the experiments was made from a Milli- $Q$ water purification system (Millipore, Bedford, MA).

The composition of normal Tyrode's solution was as follows (in $\mathrm{mM}$ ): $\mathrm{NaCl} 136.5, \mathrm{KCl} 5.4, \mathrm{CaCl}_{2} 1.8$, $\mathrm{MgCl}_{2}$ 0.53, glucose 5.5, and HEPES-NaOH buffer 5.5 (pH 7.4). To record hyperpolarization-elicited currents, the patch pipette was generally filled with a solution (in mM): $\mathrm{K}$-aspartate $130, \mathrm{KCl} 20, \mathrm{KH}_{4} \mathrm{PO}_{4} 1, \mathrm{MgCl}_{2} 1$, $\mathrm{Na}_{2} \mathrm{ATP} 3$, $\mathrm{Na}_{2} \mathrm{GTP} 0.1$, EGTA 0.1, and HEPES-KOH buffer 5 (pH 7.2). To preclude the possible contamination of $\mathrm{Cl}^{\prime}$ currents [22], $\mathrm{Cl}$ ions inside the pipette solution was replaced with aspartate. In some set of experiments, $\mathrm{GH}_{3}$ cells were incubated with PALCAR $(3 \mu \mathrm{M})$ or isoflurane $(2.0 \%[\mathrm{v} / \mathrm{v}])$ for two hours.

\section{Cell Preparations}

$\mathrm{GH}_{3}$ pituitary tumor cells, obtained from the Bioresources Collection and Research Center ([BCRC60015]; Hsinchu, Taiwan), were routinely maintained at a density of $10^{6} / \mathrm{ml}$ in Hams's F-12 nutrient media supplemented with $15 \%$ horse serum, $2.5 \%$ fetal calf serum and $2 \mathrm{mM} \mathrm{L-glutamine} \mathrm{in} \mathrm{a} \mathrm{humidified}$ environment of $5 \% \mathrm{CO}_{2} / 95 \%$ air. The murine macrophage cell line, RAW 264.7 , was obtained from the American Type Culture Collection (TIB-71; Manassas, VA). RAW 264.7 cells were grown in plastic disks containing Dulbecco's modified Eagle's medium supplemented with $10 \%$ heat-inactivated fetal bovine serum [23]. The electrophysiological experiments were commonly performed 5 or 6 days after cells had been cultured (60-80\% confluence). The colorimetric method was commonly used in examining the cell densities in microtiter plates with a tetrazolium salt (4-[3-(4-iodophenyl)-2-(4-nitrophenyl)-2H-5terasolio]-1,3-benzene disulfonate; WST) and an ELISA reader (Dynatch, Chantilly, VA) [24].

\section{Electrophysiological Measurements}

$\mathrm{GH}_{3}$ or RAW 264.7 cells used for the experiments were dissociated and an aliquot of cell suspension was immediately transferred to a recording chamber positioned on the stage of an inverted CKX-41 microscope (Olympus, Tokyo, Japan). Cells were bathed at room temperature $\left(25^{\circ} \mathrm{C}\right)$ in normal Tyrode's solution containing $1.8 \mathrm{mM} \mathrm{CaCl}_{2}$. Patch electrodes were made from Kimax-51 capillaries (\#34500; Kimble Glass, Vineland, NJ) using either a PP-830 puller (Narishige, Tokyo, Japan) or a P-97 Flaming/Brown micropipette puller (Sutter; Novato, CA). They had a resistance of 3-5 $\mathrm{M} \Omega$ when filled with different pipette solutions described above. Ion currents were measured with glass pipettes in whole-cell configuration of standard patch-clamp technique by means of an RK-400 amplifier (Bio-Logic, Claix, France) $[9,11]$.

The signals consisting of potential and current traces were stored online in a TravelMate-6253 laptop computer (Acer, Taipei, Taiwan) at $10 \mathrm{kHz}$ through a Digidata-1322A interface (Molecular Devices, Sunnyvale, CA). The latter device was equipped with an Adaptec SlimSCSI card (Milpitas, CA) via a PCMCIA slot and controlled by pCLAMP 9.2 (Molecular Devices). Current signals were low-pass filtered at $3 \mathrm{kHz}$. The voltage-step profiles generated by pCLAMP were applied to evaluate the current-voltage relationships for ion currents (e.g., $I_{\text {MEP }}$ ). Current signals were commonly analyzed using either Origin 8.0 (OriginLab, Northampton, MA) or custom-made macros built in Excel 2007 spreadsheet running under Windows-7 (Microsoft, Redmond, WA). When current signals elicited by membrane hyperpolarization from $-80 \mathrm{mV}$ were greater than $100 \mathrm{pA}$ and the corresponding standard deviation (SD) was greater than $100 \mathrm{pA}, I_{\text {MEP }}$ was identified.

\section{Concentration-Response Analysis}

Concentration-response data for PALCAR-induced stimulation of $I_{\text {MEP }}$ emerging from $\mathrm{GH}_{3}$ cells were fitted with a Hill function. That is,

$$
y=\left(E_{\max } \times[C]^{n_{H}}\right) /\left(E C_{50}^{n_{H F}}+[C]^{n_{H H}}\right)
$$


where $y$ is the relative amplitude of $\left.I_{\text {MEP; }} ; \mathrm{C}\right]$ is the PALCAR concentration; $\mathrm{IC}_{50}$ and $\mathrm{n}_{\mathrm{H}}$ are the concentration required for a $50 \%$ increase and the Hill coefficient, respectively. The non-linear least-squares fitting was made in the Solver-add-in bundled with Microsoft Excel 2007 [9, 11, 25]. The $I_{\text {MEP }}$ amplitudes were generally taken about $2 \mathrm{~min}$ after the voltage protocol was repetitively applied to the cell. The latency to $I_{\text {MEP }}$ generation is Gaussian normal distribution with mean value of $68 \pm 7 \mathrm{sec}$ when cells were repetitively hyperpolarized from -80 to $-200 \mathrm{mV}$ [11].

\section{Principal Component Analysis (PCA)}

PCA is recognized to be a widely used method in data analysis and compression $[13,14]$. It is a simple nonparametric method that allows the extraction of relevant data from mixed datasets. Mathematically, the data of current signals were exported to a transform matrix $D$ of $m$ rows (number of measurement types) and $n$ columns (number of samples) with $s$ being the datum points.

$$
D=\left[\begin{array}{ccc}
s_{1 \times 1} . & \ldots . ., & s_{1 \times n} \\
\downarrow & & \\
s_{m \times 1}, & \ldots . . ., & s_{m \times n}
\end{array}\right],
$$

For each $n, u$ is a unit vector. $\mathrm{MM}^{T} u=\lambda u$ with the largest eigenvalue being $\lambda_{1}$. The eigenvalue $\lambda_{1}$ is called the principal eigenvalue and represents the characteristics of current signals because the first component extracted in a PCA explains as much of the variability in the data as possible. Consequently, based on PCA transformation, it is convenient to change the great number of input data (i.e., a matrix of multivariate data) into a set of components (hypothetical variables) according to their importance.

The set of principal eigenvalues generated in this study were normalized by dividing all principal eigenvalues by the maximal principal eigenvalue discovered. This allows for the relative comparisons to be made. Before current signals were analyzed, white or Gaussian noise with a duration of 300 msec was created and testified for the reliability of our measures. Principal eigenvalues of greater than 0.8 were considered large, while those for white or Gaussian noise were computed to be around 0.6 and no extreme principal eigenvalues could be clearly demonstrated.

For real-time analysis of the current signals at $10 \mathrm{sec}$ prior to the start of initial $I_{\text {MEP }}\left(I_{\text {Pre }}\right)$, the digital data for each period (i.e., $5 \mathrm{msec}$ ) were taken as inputs into the transform matrix described above. The resultant principal eigenvalues representing the principal characteristic of current signals for this period were then computed. In this way, about 55 principal eigenvalues were extracted for each frame of current signal, when capacitative current transients ranging usually between the twentieth and thirtieth millisecond after the beginning of voltage pulses were removed.

The data are presented as means \pm standard error of mean (SEM) with sample sizes (n), indicating the number of cells examined. The paired or unpaired Student's $t$ test and one-way analyses of variance with a least-significance difference method for multiple-group comparisons were used for the statistical evaluation of difference among means. To evaluate the sum of squared residuals (SSR) value as a function of $\mathrm{EC}_{50}$ value for the stimulatory action of PALCAR on $I_{\text {MEP }}$ the $95 \%$ confidence interval was estimated with the aid of Fisher's $F$ distribution $[9,25]$. The PCA computations were performed using PROC FACTOR procedure in SAS 9.3 application program (SAS Institute Inc., Cary, NC). Differences were considered statistically significant at $P<0.05$.

\section{Results}

\section{Analysis of Hyperpolarization-Induced Currents in Pituitary $\mathrm{GH}_{3}$ Cells}

By use of standard whole-cell voltage-clamp recordings, we measured hyperpolarizationinduced currents existing in pituitary $\mathrm{GH}_{3}$ cells for up to $5 \mathrm{~min}$. In order to evaluate the characteristics of current signals in response to membrane hyperpolarization in these cells, the principal component analysis (PCA) was thereafter applied to current signals for calculation of the principal eigenvalues $\left(\lambda_{1}\right)$. In these experiments, immediately after a stable whole-cell model was achieved, membrane hyperpolarizations at a rate of $0.1 \mathrm{~Hz}$ were repetitively applied from a holding potential of $-80 \mathrm{mV}$ to $-200 \mathrm{mV}$. Figure 1 depicts a typical 


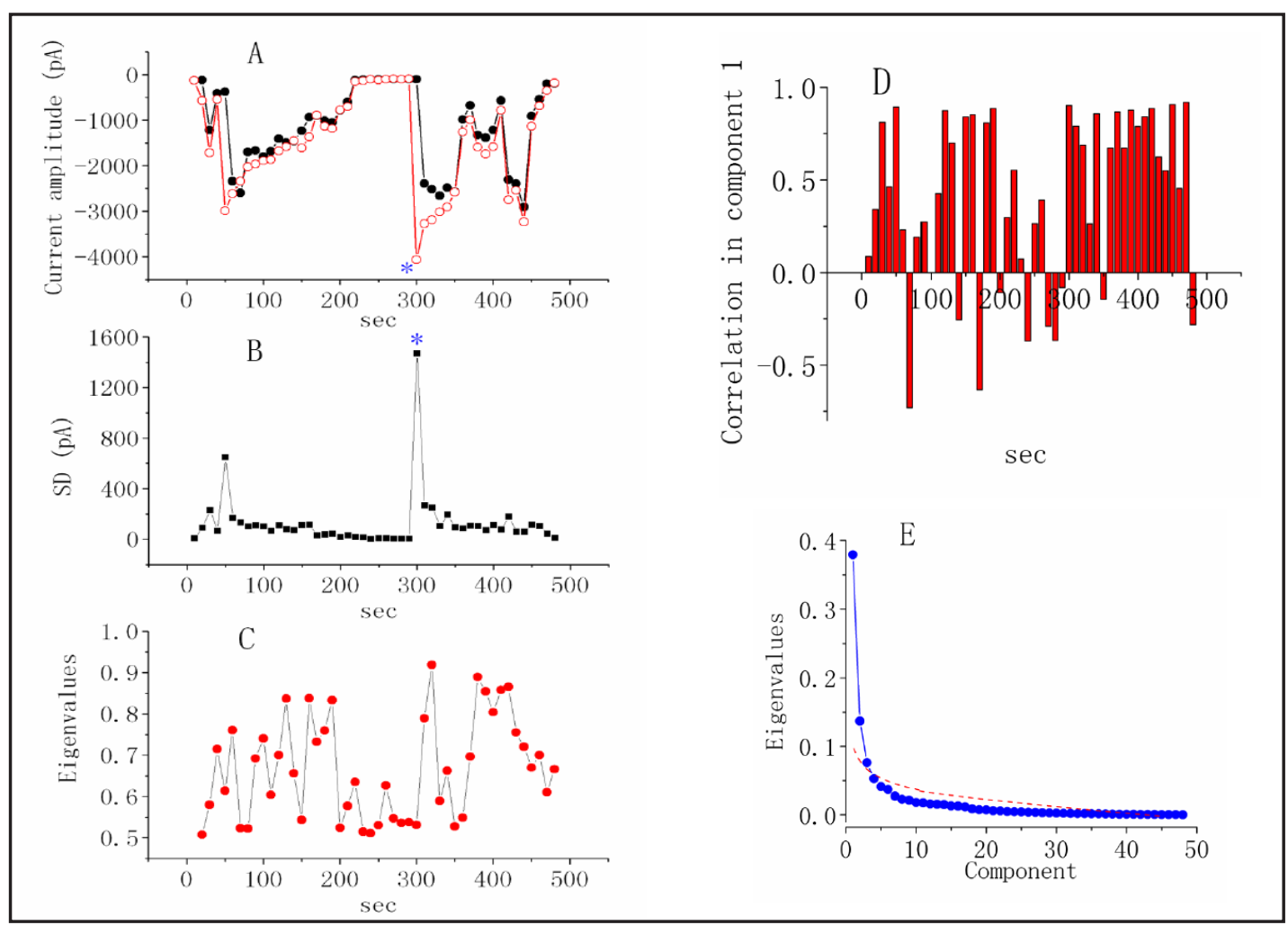

Fig. 1. Analyses of hyperpolarization-induced currents recorded from pituitary $\mathrm{GH}_{3}$ cells. In these experiments, cells were bathed in $\mathrm{Ca}^{2+}$-free Tyrode's solution containing $10 \mathrm{mM} \mathrm{CsCl}$. CsCl was used to prevent the contamination of whole-cell $\mathrm{K}^{+}$currents. The $I_{\text {MEP }}$ was elicited in an episodic fashion as the cell examined was hyperpolarized from -80 to $-200 \mathrm{mV}$ with a duration of $300 \mathrm{msec}$ at a rate of $0.1 \mathrm{~Hz}$. (A) Time course of changes in current amplitude, standard deviation (SD) of corresponding amplitude (B), and principal eigenvalues (C) of current signals. The measurement was made immediately after wholecell configuration was established. Red open circles and black filled circles in (A) correspond to current amplitudes measured at the beginning $(10 \mathrm{msec})$ and end $(295 \mathrm{msec})$ of repetitive hyperpolarizations to $-200 \mathrm{mV}$, respectively. Black filled squares in (B) correspond to SD of current amplitude, and red filled circles in (C) indicate the principal eigenvalues derived from PCA. (D) Bar graph showing the correlation coefficient between each signal and the first component versus time. Correlation coefficient in each pulse was made in the first component of PCA. On note, changes in the positive correlation coefficient tend to be coincident with those in principal eigenvalues. (E) Scree plot (i.e., simple plot of eigenvalues) of hyperpolarizationinduced inward currents which shows the proportion of variance for each principal component. The red dashed curve indicates the eigenvalues expected under a random model for separating the meaningful components from the trivial components. Thus, the eigenvalues under this red curve may represent nonsignificant components. Note that there are three components (above the red curve) representing the most important ones extracted in PCA.

example of one of these experiments showing effects of large hyperpolarization on membrane current measured from a pituitary $\mathrm{GH}_{3}$ cell. Consistent with previous observations [10-12, 26], a population of irregular and transient inward currents occurring in a periodical fashion were clearly elicited. These inward currents occurring asynchronously were increased with greater hyperpolarizations and have been previously identified as $I_{\mathrm{MEP}}$. The time courses of changes in the amplitude, standard deviation (SD) and principal eigenvalue of these currents measured at the potential of $-200 \mathrm{mV}$ are illustrated in Fig. 1. Notably, when current signals elicited by repetitive hyperpolarizations were pooled and transformed with PCA, the larger principal eigenvalues which are assumed to be greater than 0.8 were found to coincide with the emergence of large inward currents. As the $I_{\mathrm{MEP}}$ became declined, principal eigenvalues 


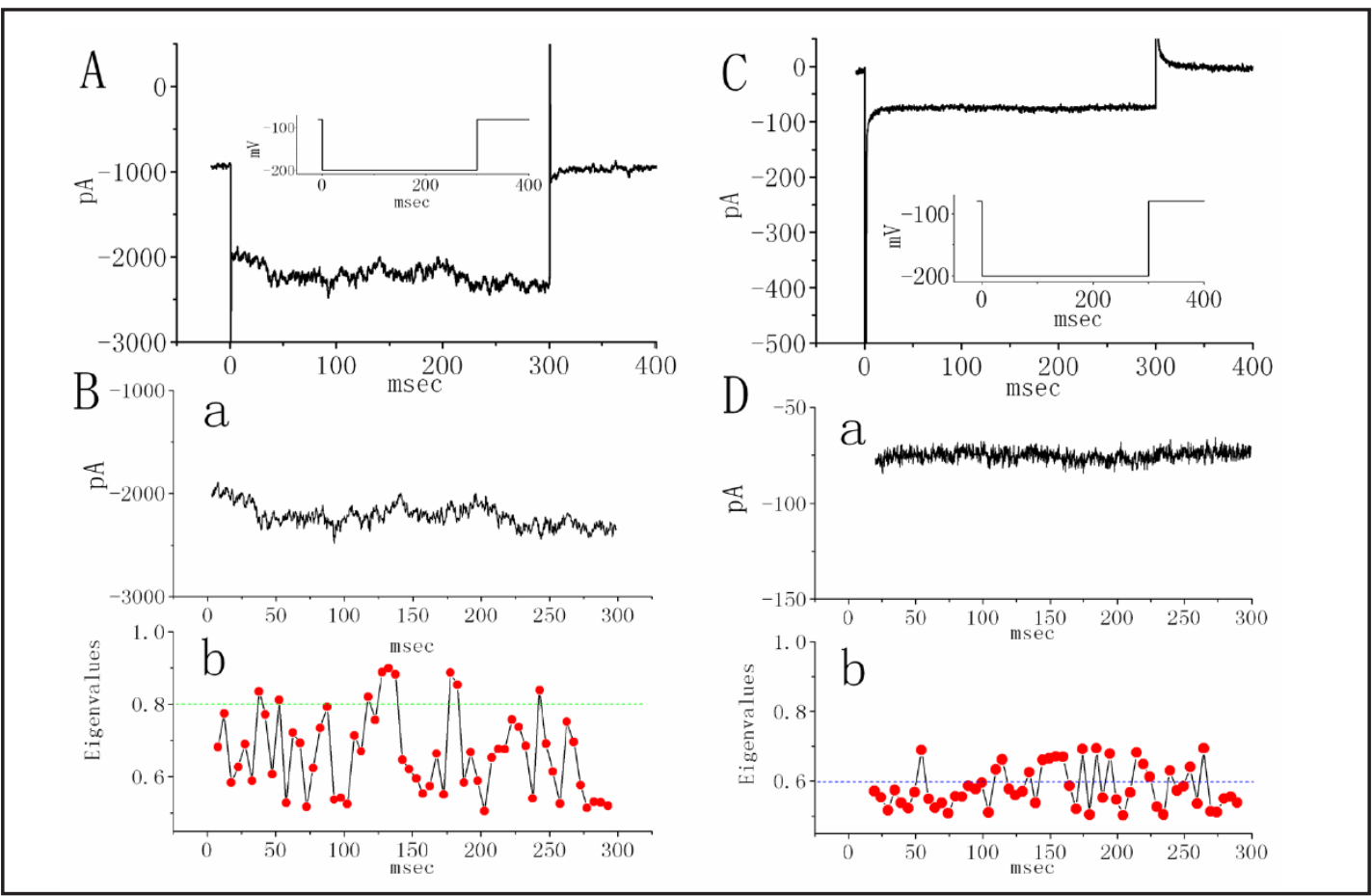

Fig. 2. Analyses of $I_{\mathrm{MEP}}$ recorded from pituitary $\mathrm{GH}_{3}$ cells. (A) Original $I_{\mathrm{MEP}}$ trace corresponding to the data indicated by asterisk in Fig. 1B (at $300 \mathrm{msec}$ ). The current signal was elicited by hyperpolarization to $-200 \mathrm{mV}$ from $-80 \mathrm{mV}$. Panel $(\mathrm{Ba})$ is original current trace at the potential of $-200 \mathrm{mV}$, whereas panel (Bb) represents the time course of principal eigenvalues derived from current signal in $(\mathrm{Ba})$. The dashed green line in (Bb) indicates the principal eigenvalue of 0.8. (C) Original $I_{\mathrm{MEP}}$ obtained in the presence of $\mathrm{LaCl}_{3}(100$ $\mu \mathrm{M})$. Panel $(\mathrm{Da})$ was obtained in the presence of $\mathrm{LaCl}_{3}(100 \mu \mathrm{M})$, whereas panel $(\mathrm{Db})$ represents the time course of resultant principal eigenvalues from $(\mathrm{Da})$. The dashed blue line in $(\mathrm{Db})$ indicates the principal eigenvalue of 0.6 . Inset in (A) and (C) indicates the voltage protocol used. Notably, as cells were exposed to $\mathrm{LaCl}_{3}$, besides that the amplitude of hyperpolarization-induced current was greatly decreased, the resultant principal eigenvalues derived from these current signals became diminished and was around 0.6 (indicated by the blue dashed line), suggesting that no clear extreme principal eigenvalues can be demonstrated in the presence of $\mathrm{LaCl}_{3}$.

derived from PCA were greatly reduced to be around 0.5. It is noted that large principal eigenvalues of greater than 0.8 (in a normalized set) are closely linked to the large deflections of $I_{\text {MEP }}$ (indicated by asterisk in Fig. 1B). Similarly, changes in correlation coefficient of the first principal component extracted in a PCA (Fig. 1D) tended to coincide with clear extreme principal eigenvalues in Fig. 1C. Similar results were obtained in 17 different cells examined. The correlation $(\mathrm{r})$ between changes in the correlation coefficient of the first principal component extracted and the extreme principal eigenvalue is $0.78 \pm 0.02(n=17)$. Based on the scree plot by PCA conducted on hyperpolarization-elicited current signals (Fig. 1E), there exhibit to be the first three principal components which are well retained owing to a fairly large size of the eigvenvalues directly related to these ones. Notably, the magnitude of the eigenvalues falls off quickly, such that about $70 \%$ of the value is contained in the first three eigenvectors which tend to account for a large proportion of the variability. This implies that a good approximation of the full matrix derived from current signals in response to membrane hyperpolarization can be computed using only a subset of the eigenvectors and eigenvalues.

Analysis of $I_{M E P}$ Signals from $\mathrm{GH}_{3}$ Cells

We further attempted to examine whether or not PCA can fairly discriminate the MEPassociated currents elicited by each pulse on the basis of the characteristics of principal 
Fig. 3. Real-time analysis of $I_{\text {Pre }}$ signal in response to membrane hyperpolarization. (A) Original trace of $I_{\text {Pre }}$ (i.e., hyperpolarization-induced current occurring $10 \mathrm{sec}$ prior to the start of initial $I_{\text {MEP }}$ ) in a $\mathrm{GH}_{3}$ cell was shown. Inset in (A) indicates the voltage protocol used. (B) $I_{\text {Pre }}$ signal at the potential of $-200 \mathrm{mV}$. In inset of (B), blue current trace was amplified from (B) (indicated by dashed box). Note that there were minuscule inward currents $\left(I_{\min }\right)$ immediately before the rise of large inward current indicated by a large downward deflection. (C) Principal eigenvalues using PCA applied to $I_{\mathrm{Pre}}$ signal derived from (B). Note that there are two

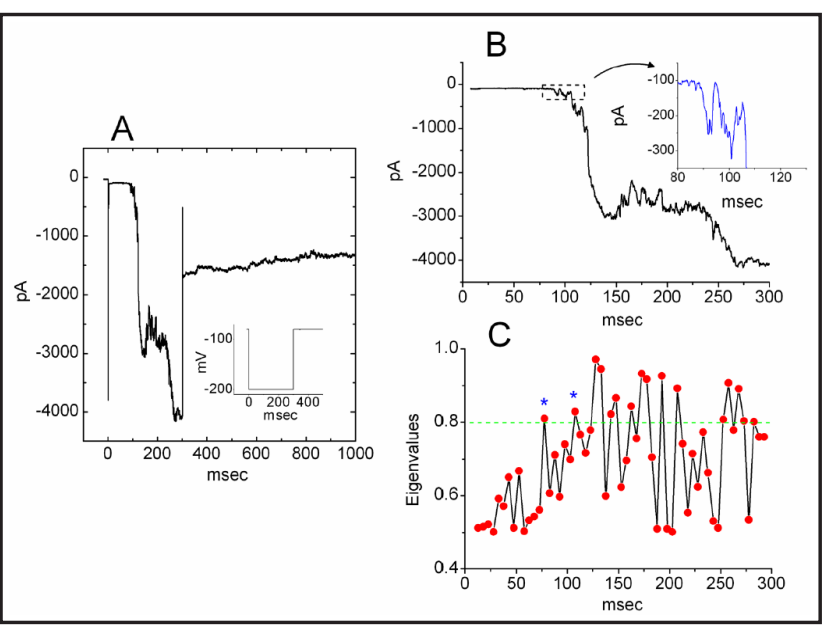

clear extreme principal eigenvalues indicated by asterisk in (C) which coincide with the occurrence of herald minuscule inward currents $\left(I_{\min }\right)$ prior to the rise of large MEP-associated inward current. The green dashed line indicates the principal eigenvalue at the level of 0.8 .

eigenvalues. Previous studies have shown the ability of $\mathrm{LaCl}_{3}$ or $\mathrm{MnCl}_{2}$ to suppress $I_{\text {MEP }}[9$, 11]. The single $I_{\text {MEP }}$ elicited by large hyperpolarization with subsequent addition of $\mathrm{LaCl}_{3}$ or $\mathrm{MnCl}_{2}$ was then analyzed on a real-time basis. As shown in Fig. 2, as current signal elicited by large hyperpolarization was elicited, the corresponding principal eigenvalues extracted in PCA were found to be progressively elevated over time. Multiple peaks with principal eigenvalues of greater than 0.8 can be noted (Fig. 2Bb). A subsequent application of $\mathrm{LaCl}_{3}$ $(100 \mu \mathrm{M})$ to cells, the $I_{\text {MEP }}$ magnitude was considerably reduced (Fig. 2), as evidenced by the significant decrease in current amplitude to $203 \pm 12 \mathrm{pA}(\mathrm{n}=12, P<0.01)$ from a control value of $2317 \pm 245 \mathrm{pA}(\mathrm{n}=12)$. Accordingly, the principal eigenvalues extracted in PCA from such current signal over time were significantly declined to be around 0.6 (Fig. 2Db). Similar results were also obtained as cells were exposed to $\mathrm{MnCl}_{2}(100 \mu \mathrm{M})$. Subsequent application of $\mathrm{LaCl}_{3}$ or $\mathrm{MnCl}_{2}$ to $\mathrm{GH}_{3}$ cells can not only decrease $I_{\text {MEP }}$ amplitude, but also diminish principal eigenvalue extracted in PCA. These interesting results suggest that the extreme principal eigenvalues derived from current signals are well correlated with the emergence of $I_{\text {MEP }}$ elicited by large hyperpolarizations.

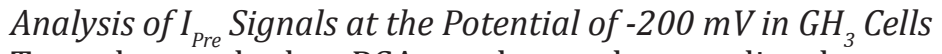

To evaluate whether PCA can be used to predict the appearance of MEP-associated currents, the current signals at 10 sec preceding the start of $I_{\text {MEP }}$ rise (i.e., $I_{\text {Pre }}$ ) which were accompanied by the elevated SD of current amplitude were further analyzed. As shown in Fig. 3B and 3C, the escalating rise of current amplitudes indicated in a downward deflection tends to be coincident with the increased principal eigenvalues. Interestingly, concomitant with occurrence of herald minuscule inward currents $\left(I_{\min }\right)$, the extreme principal eigenvalues as denoted by asterisks in Fig. 1B were also noted to precede the abrupt emergence of $I_{\text {MEP }}$ in response to membrane hyperpolarization, although the amplitude of $I_{\min }$ was small and ranged between 100 and $300 \mathrm{pA}$. The results reflect that PCA is effective at distinguishing MEP-induced currents from randomly noisy ones.

Effects of Changes in Membrane Polarization on the Principal Eigenvalues of $I_{\text {Pre }}$ Signals

Previous analysis with detrended fluctuation analysis showed that the scaling exponent of $I_{\text {MEP }}$ signals remained unaffected by the magnitude of membrane hyperpolarization [11]. We further evaluated if the level of membrane hyperpolarization can influence $I_{\text {Pre }}$ or the principal eigenvalues of this current signals. As illustrated in Fig. 4, when the cell was hyperpolarized to $-160 \mathrm{mV}$ from a holding potential of $-80 \mathrm{mV}$, multiple runs of $I_{\min }$ with a 
Fig. 4. Effect of different membrane hyperpolarizations on $I_{\min }$ and corresponding principal eigenvalues. (A) Original $I_{\text {Pre }}$ trace obtained as the hyperpolarization pulse from -80 to $-160 \mathrm{mV}$ was applied. Inset in $(\mathrm{A})$ indicates the voltage protocol used. (B) $I_{\text {pre }}$ signal obtained at the potential of $-160 \mathrm{mV}$. Blue current trace in inset was amplified from (B) (indicated by the dashed box). (C) Time course in changes of principal eigenvalue of an $I_{\text {Pre }}$ signal derived from (B) which was measured at -160 $\mathrm{mV}$. The asterisks in (C) indicate the extreme principal eigenvalue before the rise of large current amplitude. (D) Summary of the data depicting effect of membrane potentials on duration of $I_{\min }$ (mean \pm SEM, $\mathrm{n}=6$ for each bar). The duration of herald $I_{\min }$ elicited by membrane hyperpolarization was taken to be the time ranging between the initial appearance of extreme principal eigenvalue and the large rise in current amplitude

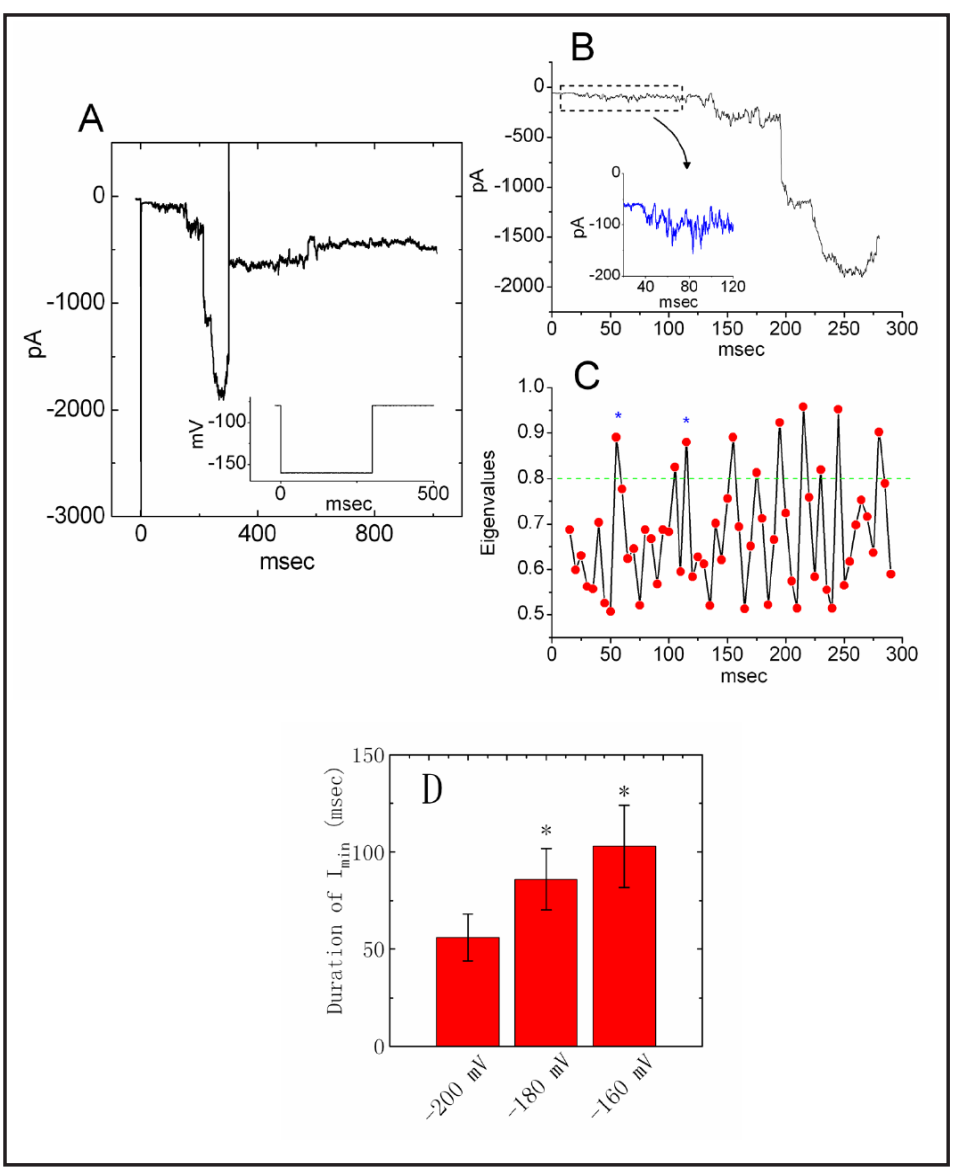

(i.e., greater than $500 \mathrm{pA}$ ). Notably, under our whole-cell recordings, with greater membrane hyperpolarizations, the duration for $I_{\min }$ occurrence became progressively shortened. *Significantly from the values at $-200 \mathrm{mV}(P<0.05)$.

Fig. 5. Characterization of $I_{\mathrm{Pre}}$ and corresponding principal eigenvalues in isoflurane-treated $\mathrm{GH}_{3}$ cells. In this set of experiments, $\mathrm{GH}_{3}$ cells were treated with isoflurane $(2.0 \%)$ for two hours. (A) Original $I_{\text {Pre }}$ trace at $-200 \mathrm{mV}$ measured from an isofluranetreated $\mathrm{GH}_{3}$ cell. (B) Principal eigenvalues of $I_{\text {Pre }}$ signal derived from $(\mathrm{A})$. The green dashed line indicates the principal eigenvalue at the level of 0.8 . Note that, in cells exposed to isoflurane, the duration of herald $I_{\min }$ in response to membrane hyperpolarization remained unaltered, although the demarcation line between $I_{\min }$ and the rise of large current amplitude becomes slightly unclear.

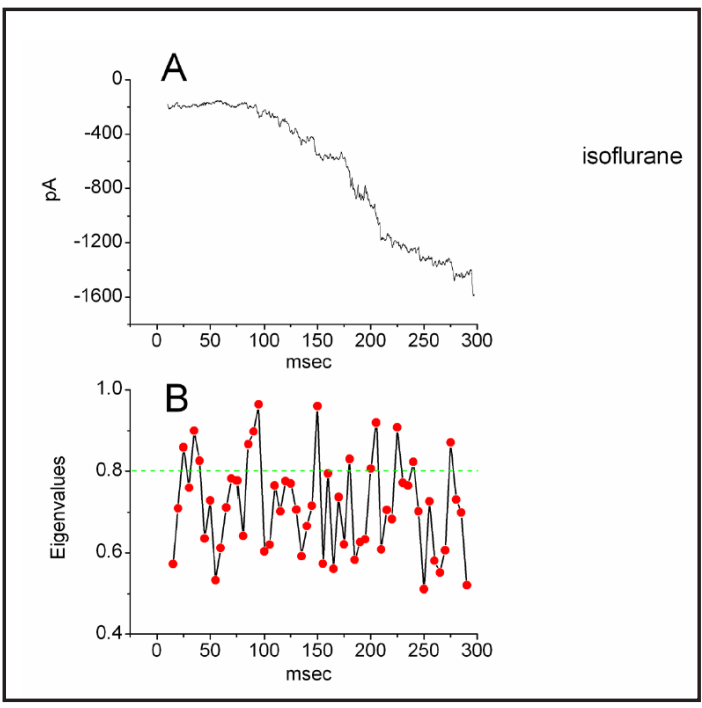

longer duration tended to appear before large rise in downward deflection. Additionally, when the membrane became more hyperpolarized, the duration of both heralded $I_{\min }$ emergence and principal eigenvalues existing higher values was significantly shortened (Fig. 4D). For example, the duration of $I_{\min }$ measured at $-200 \mathrm{mV}$ was $56 \pm 12 \mathrm{msec}(\mathrm{n}=6)$ 
Fig. 6. Characterization of $I_{\mathrm{Pre}}$ and corresponding principal eigenvalues in PALCAR-treated $\mathrm{GH}_{3}$ cells. In this set of experiments, $\mathrm{GH}_{3}$ cells were treated with PALCAR $(3 \mu \mathrm{M})$ for two hours. (A) Original $I_{\text {Pre }}$ trace from a $\mathrm{GH}_{3}$ cell. The upper part of (A) indicated the voltage protocol used. (B) $I_{\text {Pre }}$ signal at $-120 \mathrm{mV}$. Blue current signal in Inset was amplified from (B) (indicated by the dashed box). (C) Corresponding principal eigenvalues of $I_{\text {Pre }}$ signal. Notably, the amplitude of herald $I_{\text {min }}$ tends to be smaller together with no clear extreme principal eigenvalues which were less than 0.8 .

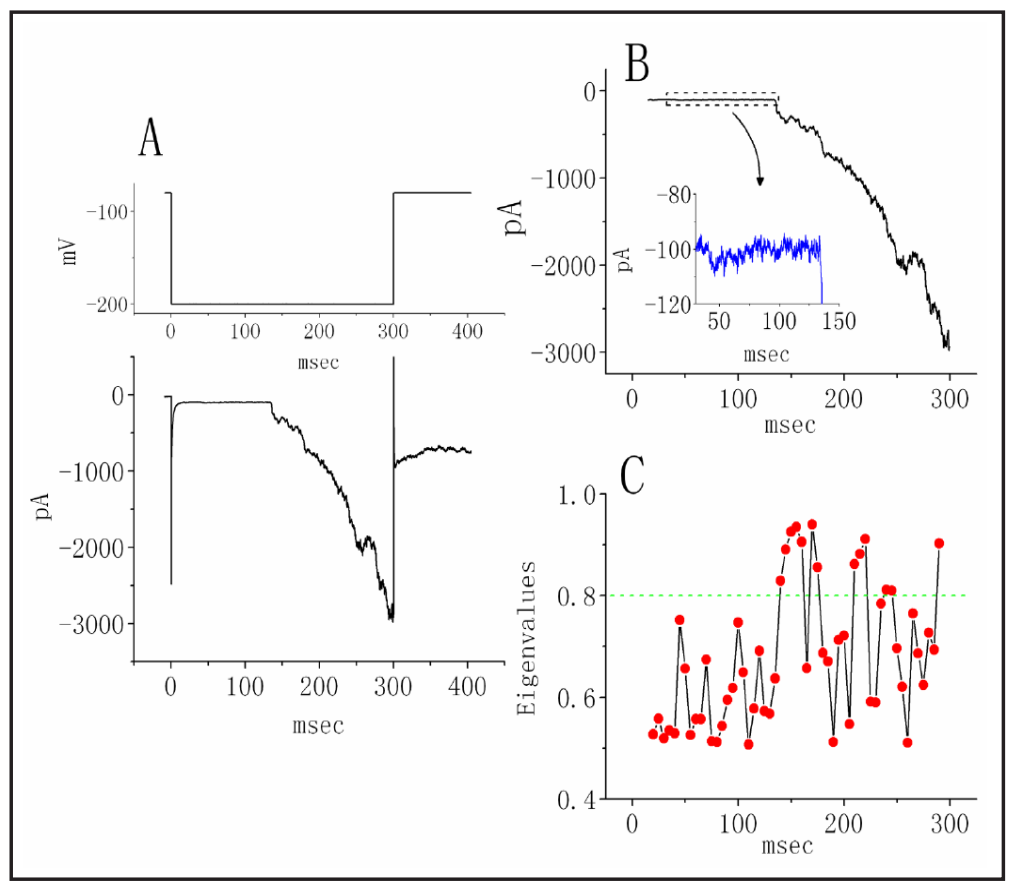

which was significantly shorter than that at $-160 \mathrm{mV}(103 \pm 21 \mathrm{msec}[\mathrm{n}=6])$. The frequency of extreme principal eigenvalues greater than 0.8 during $I_{\text {min }}$ occurrence was noted to increase as membrane became more hyperpolarized. The results demonstrate that, unlike the scaling exponent of $I_{\text {MEP }}$ signals [11], the duration of both $I_{\min }$ and corresponding higher principal eigenvalues may rely on the different levels of membrane hyperpolarization.

Analysis of $I_{P r e}$ Signals Recorded from Isoflurane-Treated $\mathrm{GH}_{3}$ Cells

In the next set of experiments, we tested if inhalational anesthetics isoflurane can alter the biophysical properties of $I_{\mathrm{MEP}}$. In these experiments, $\mathrm{GH}_{3}$ cells were pretreated with 2.0 $\%(\mathrm{v} / \mathrm{v})$ isoflurane for two hours. Membrane hyperpolarizations under the same protocol described above were repetitively applied to the cell and PCA was used for analyzing the principal eigenvalues. As shown in Fig. 5, the heralded $I_{\min }$ together with the large principal eigenvalues was clearly shown. The results suggest that pretreatment of cells with isoflurane did not significantly alter both duration of heralded $I_{\min }$ and generation of extreme principal eigenvalues extracted in PCA, although this agent may influence the biophysical properties of membrane ion channels [27].

\section{Analysis of $I_{\text {Pre }}$ Signals Recorded from PALCAR-Treated $\mathrm{GH}_{3}$ Cells}

Previous work has demonstrated that PALCAR could reduce electrophoretic mobility of erythrocytes [16], elevate intracellular $\mathrm{Ca}^{2+}$ in vascular endothelial cells [20] and increase the amplitude of late $\mathrm{Na}^{+}$current [21]. Therefore, we further investigated the biophysical properties of $I_{\mathrm{MEP}}$ in cells exposed to PALCAR $(3 \mu \mathrm{M})$ for 2 hours. Interestingly, as membrane hyperpolarization was applied to the cell, the $I_{\text {Pre }}$ signal emerging before the rise of initial $I_{\text {MEP }}$ was not noted to exhibit the presence of heralded $I_{\text {min }}$ (Fig. 6). Moreover, the principal eigenvalues derived from $I_{\mathrm{Pre}}$ did not show the value greater than 0.8 in current signals immediately prior to the escalating inward current elicited by membrane hyperpolarization.

Stimulatory Effect of PALCAR on $I_{M E P}$ in Pituitary $\mathrm{GH}_{3}$ Cells

Because the characteristics of $I_{\text {MEP }}$ were altered in PALCAR-treated cells, we next explored whether PALCAR could directly modify the magnitude of hyperpolarization-induced $I_{\text {MEP }}$ in $\mathrm{GH}_{3}$ cells. In these experiments, whole-cell configuration was used to investigate electrical 


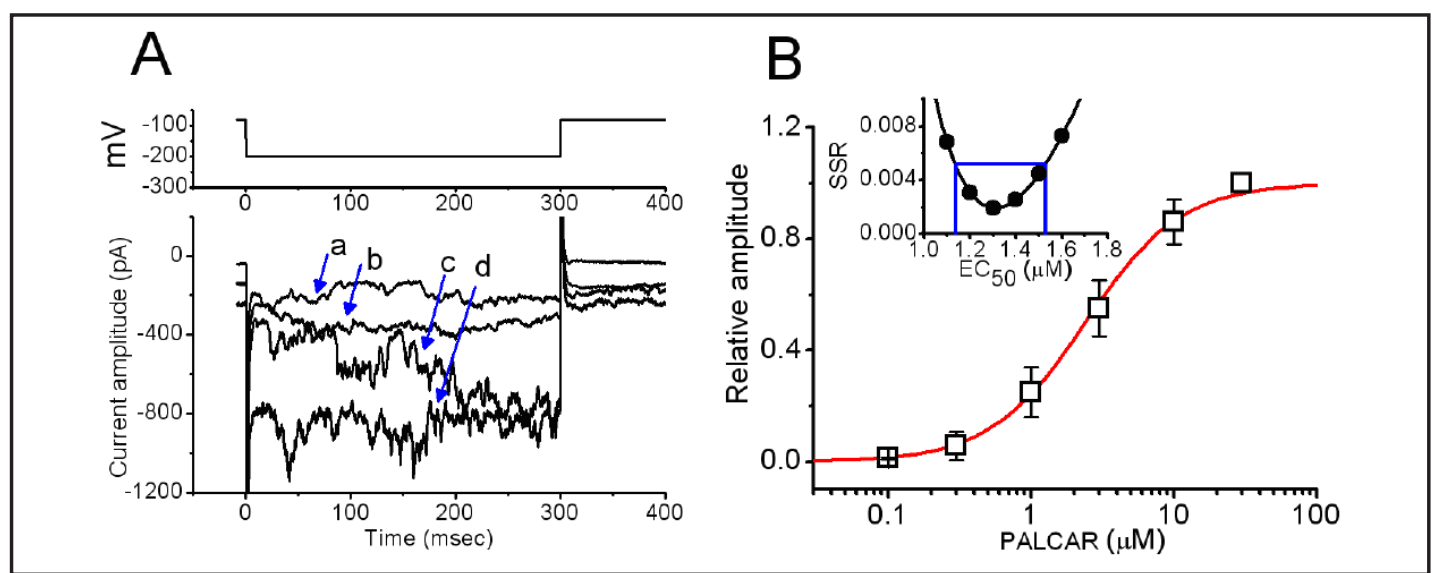

Fig. 7. Stimulatory effect of PALCAR on $I_{\mathrm{MEP}}$ in pituitary $\mathrm{GH}_{3}$ cells. In these experiments, cells were bathed in $\mathrm{Ca}^{2+}$-free Tyrode's solution containing $10 \mathrm{mM} \mathrm{CsCl}$. The cells were held at $-80 \mathrm{mV}$ and hyperpolarizing pulses to $-200 \mathrm{mV}$ with a duration of $300 \mathrm{msec}$ at a rate of $0.05 \mathrm{~Hz}$ were applied. (A) Superimposed current traces in response to membrane hyperpolarization. a: control; b: $1 \mu \mathrm{M}$ PALCAR, c: $3 \mu \mathrm{M}$ PALCAR; d: 10 $\mu \mathrm{M}$ PALCAR. The upper part of (A) indicates the voltage protocol used. (B) The relationship between the relative amplitude of $I_{\text {MEP }}$ and the PALCAR concentration. At -200 mV, current amplitude in the presence of $30 \mu \mathrm{M}$ PALCAR was considered to be 1.0. The smooth line represents the best fit to the Hill function as described in "Materials and methods". The $\mathrm{EC}_{50}$ value and Hill coefficient for PALCAR-induced stimulation of $I_{\mathrm{MEP}}$ was $2.4 \mu \mathrm{M}$ and 1.3, respectively. Each point represents the mean $\pm \mathrm{SEM}(\mathrm{n}=7-13)$. The inset in (B) shows confidence assessment of best-fit parameter values. The parameter range corresponds to the approximate 95\% confidence intervals. Gray line marks parameter value (i.e., $\mathrm{EC}_{50}$ ) at which the sum of squared residuals (SSR) amounts to 0.0052 (see text).

properties of macroscopic $I_{\text {MEP }}$ with or without the addition of different concentrations of PALCAR. Similar to the experimental profile described above, cells were bathed in $\mathrm{Ca}^{2+}$-free Tyrode's solution. When the cell was held at $-80 \mathrm{mV}$, the hyperpolarizing pulse from -80 to $-200 \mathrm{mV}$ with a duration of $300 \mathrm{msec}$ was applied. As shown in Fig. 7A, as cells were exposed to different concentrations of PALCAR, the $I_{\text {MEP }}$ amplitude was greatly increased (traces b to $d$ in Fig. 7A). For example, at the potential of $-200 \mathrm{mV}$, PALCAR $(3 \mu \mathrm{M})$ significantly increased the $I_{\text {MEP }}$ amplitude from $245 \pm 34$ to $657 \pm 67 \mathrm{pA}(\mathrm{n}=8, P<0.05)$. After washout of the compound, the current amplitude partially returned to $425 \pm 38 \mathrm{pA}(\mathrm{n}=6)$. When $\mathrm{K}^{+}$ions in the pipette solution were replaced with equimolar concentrations of $\mathrm{NMDG}^{+}$, this current could still be elicited by PALCAR $(3 \mu \mathrm{M})$, although the $I_{\mathrm{MEP}}$ amplitude became relatively smaller. Moreover, PALCAR-elicited increase of $I_{\mathrm{MEP}}$ in $\mathrm{GH}_{3}$ cells was significantly reversed by subsequent addition of $\mathrm{LaCl}_{3}$ or $\mathrm{MnCl}_{2}$, but not by iberiotoxin $(200 \mathrm{nM})$, apamin $(200 \mathrm{nM})$ or glibenclamide $(30 \mu \mathrm{M})$ (data not shown).

The relationship between the PALCAR concentration and the relative amplitude of $I_{\text {MEP }}$ was then derived and plotted (Fig. 7B). The half-maximal concentration required for its stimulation of $I_{\mathrm{MEP}}$ was $2.4 \pm 0.3 \mu \mathrm{M}(\mathrm{n}=13)$. In the SSR plot shown in the inset of Fig. 7B, there was a horizontal line at $\mathrm{SSR}=0.0052$ which was used to determine the two $\mathrm{EC}_{50}$ values. For a 95\% confidence interval, the lower and upper values were then computed to be 1.43 and $1.53 \mu \mathrm{M}$, respectively, indicating that the $\mathrm{EC}_{50}$ value computed is precise and significant. It is also noted that there was a steep slope on both sides of the minimum, indicating that the $\mathrm{EC}_{5}$ value for PC-stimulated $I_{\text {MEP }}$ was determined with high confidence [25]. Similarly, lysophosphatidylcholine $(10 \mu \mathrm{M})$, a glycerol-based lysophospholipid, was able to increase the $I_{\text {MEP }}$ amplitude in these cells (data not shown). Lysophosphatidylcholine may mediate its stimulatory effect on $I_{\mathrm{MEP}}$ through a pathway similar to or identical to PALCAR. However, neither tetrodotoxin nor ranolazine produced any effects on PALCAR-stimulated $I_{\text {MEP }}$ found 
Fig. 8. Characterization of $I_{\text {Pre }}$ and corresponding principal eigenvalues in RAW 264.7 macrophages. (A) Original $I_{\text {Pre }}$ trace at -200 $\mathrm{mV}$. Blue current trace in Inset was amplified from (A) (indicated by the dashed box). (B) Principal eigenvalues of $I_{\text {Pre }}$. The green dashed line indicates the principal eigenvalue at the level of 0.8 . The $I_{\min }$ along with high principal eigenvalues was noted to emerge before the start of large rise in current amplitude.

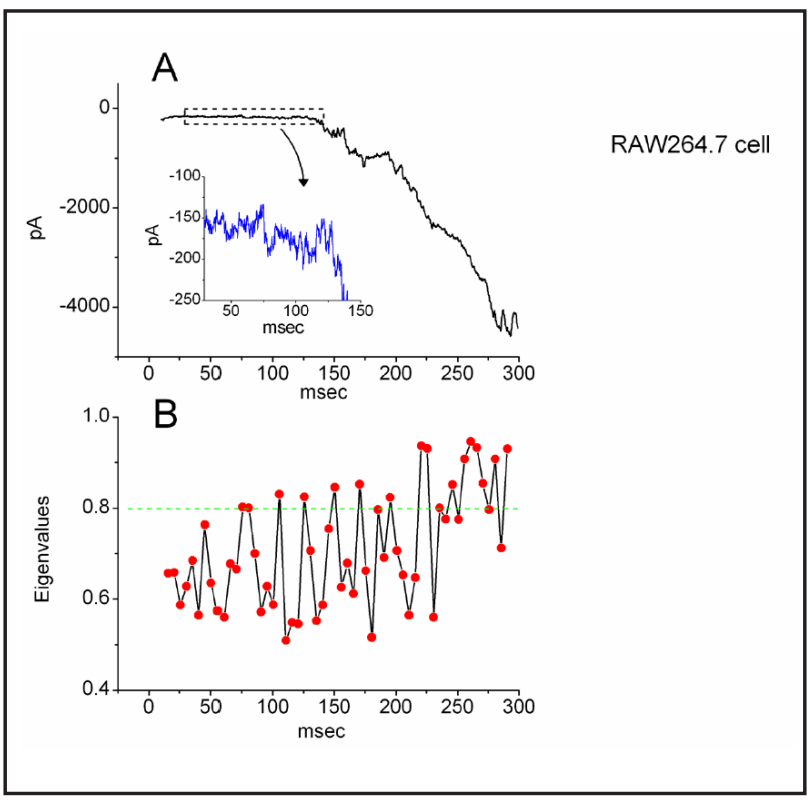

in these cells. Taken together, results from these observations lead us to propose that, in $\mathrm{GH}_{3}$ cells, PALCAR has a stimulatory effect on $I_{\mathrm{MEP}^{\prime}}$, rather than on late $\mathrm{Na}^{+}$currents $[21,28,29]$.

\section{Analysis of $I_{\text {Pre }}$ Emerging from RAW 264.7 Macrophages}

Because the occurring events of $I_{\mathrm{MEP}}$ in response to membrane hyperpolarization might vary among different types of cells [11, 12], $I_{\text {Pre }}$ measured from RAW 264.7 macrophages were further investigated and analyzed with PCA. Consistent with previous observations in RAW 264.7 cells [11], the present results clearly showed that the $I_{\mathrm{MEP}}$ can be readily elicited by repetitive large hyperpolarizations. As illustrated in Fig. 8, the heralded $I_{\min }$ existing in $I_{\text {Pre }}$ signal can be found immediately before the start of initial large inward current. As the $I_{\min }$ was extracted in PCA, the high principal eigenvalues were noted to appear prior to large downward deflection in response membrane hyperpolarization. The $I_{\min }$ duration elicited by membrane hyperpolarization to $-200 \mathrm{mV}$ from a holding potential of $-80 \mathrm{mV}$ was measured to be $39 \pm 4$ msec $(n=9)$. This value was significantly lower as compared with that from pituitary $\mathrm{GH}_{3}$ cells. These results reflect that by use of PCA, the $I_{\text {Pre }}$ signals observed in the two types of cells were consistently found to display heralded $I_{\min }$ accompanied by the appearance of extreme principal eigenvalues. The observed difference in $I_{\min }$ duration between these cells could be explained by either different elastic properties of phospholipid bilayers or later stress of the hydrocarbon chain region.

\section{Discussion}

In this study, we established an important association between times when extreme principal eigenvalues are given and the occurrence of large current amplitude rise. The heralded $I_{\min }$ together with high principal eigenvalues present in $I_{\text {Pre }}$ current signals is likely to be followed by the rise of large current amplitude. PCA is applied to hyperpolarizationinduced inward currents to detect heralded $I_{\min }$ associated with the emergence of $I_{\mathrm{MEP}}$ in $\mathrm{GH}_{3}$ and RAW 264.7 cells. Therefore, it is anticipated from this study that MEP-induced opening of membrane pores could occur earlier and be coincident with the emergence of heralded $I_{\min }$. By using PCA, we provided information not only on the process of pore formation, but also suggestive of the kinetics of pore generation related to MEP. This is potentially important because we might define the optimal conditions for the electroporation of cells, design systems for MEP, and protection against serious cell damage during this process. 
The hyperpolarization-induced activation and irregular time course observed in these cells strongly suggested that $I_{\text {MEP }}$ was produced by transient rupture of cell membrane due to changes in the electrical field associated with large hyperpolarization $[12,26]$. It is possible that repetitive application of large hyperpolarizations creates the momentum necessary to influence the phospholipid bilayers. Large hyperpolarizations from -80 to $-200 \mathrm{mV}$ cause significant current variations, which were attributable to changes in the electrical field. More importantly, PCA presented herein can not only discriminate between MEP-related current signals and background noises, but also give a mathematical representation of hyperpolarization-elicited current signals without the need for prior observational analysis, as long as we understand how such signals are characterized by principal eigenvalues. Our results also prompted us to indicate that although change in the level of membrane polarization did not alter the correlation properties of $I_{\text {Pre }}$ [11], the events of heralded $I_{\min }$ along with higher eigenvalues became larger with greater hyperpolarizations.

Compatible with earlier studies [11], our results indicate that the higher principal eigenvalues extracted in PCA are not simply due to an increase in the magnitude of $I_{\text {MEP }}$ elicited by large hyperpolarizations, but also attributable in part to some other physiological phenomena. In other words, memory may arise from the internal dynamics of MEP-induced channels and is not clearly described by Markovian processes [11]. It also needs to be noted that PCA method used in this study is effective at distinguishing the noises of large currents from the noises of small currents; however it would not an expected difference for all kinds of the noises inherent in large currents.

Previous reports showed that in response to membrane hyperpolarization, the translocation may occur on two disparate time scales, namely, the electrophoretic time $(\sim \mathrm{msec})$, and the diffusive time ( $\sim \mathrm{sec})[5]$. If the pulse is sufficiently strong and long to complete the translocation via electrophoretic drifting, then the electrophoretic time scale is observed. As the whole data were analyzed with PCA, although the emergence of $I_{\text {MEP }}$ elicited by repetitive pulses was coincident with the higher principal eigenvalues, no prediction of pore formation was demonstrated, suggesting the diffusive time $(\sim \sec )$ for MEP-associated channels could not be evaluated with PCA effectively. However, our study showed that, distinguishable from the detrended fluctuation analysis [11], PCA could be used in real-time prediction of $I_{\text {MEP }}$ emergence (i,.e., electrophoretic time) through early detection of MEPassociated current signals.

Consistent with previous studies $[10,11,26]$, the externally applied electrical field directs ion movement and influences the kinetics of $I_{\text {MEP }}$. PCA presented herein is capable of detecting high principal eigenvalues in the series of current traces elicited by membrane hyperpolarization. If this analysis is valid, findings from this study indicate that, for larger $I_{\text {MEP }}$, clear extreme principal eigenvalues generated by PCA of herald $I_{\min }$ can potentially act as the $I_{\mathrm{MEP}}$ precursors. It is also anticipated that PCA has the capacity to discern such precursors within 50-100 msec prior to the escalating rise in large current amplitude. Therefore, the present results provide the evidence to show the existence of different principal eigenvalues extracted from current signals, which might be applied to predict the initiation of $I_{\mathrm{MEP}}$ formation in different types of cells. However, whether PCA can be specific for $I_{\text {MEP }}$ remains to be further determined.

The addition of $\mathrm{LaCl}_{3}$ to the cell caused the principal eigenvalues extracted in PCA to become significantly lowered, while the $I_{\text {MEP }}$ signal elicited by membrane hyperpolarization showed multiple peaks of high principal eigenvalues. However, in isoflurane-treated $\mathrm{GH}_{3}$, the clear extreme principal eigenvalues coincident with appearance of herald $I_{\min }$ remained intact, although isoflurane may alter the activity of ion channels [27]. Moreover, the resealing of electropores induced by large membrane hyperpolarization in these cells tends to be associated with the constant lowering of eigenvalues in these cells.

A recent paper by Nesin et al. [30] reported that MEP with nanosecond pulsed electric field caused inhibition of $I_{\mathrm{Na}}$ as well as an increase in a noninactivating leak current, which was likely due to the formation of nanoelectropores or larger pores in the plasma membrane. Another study described the mechanism of electrical field-induced inhibition 
of $I_{\mathrm{Na}}$ and demonstrated that it was not mediated by electrical field-induced $\mathrm{Na}^{+}$influx or altered $\mathrm{Ca}^{2+}$ signaling in response to pulsed electrical field [28]. The PALCAR-stimulated $I_{\text {MEP }}$ observed in $\mathrm{GH}_{3}$ cells is unlikely to be due to any changes in $I_{\mathrm{Na}}$ although it was reported to alter the kinetics of $I_{\mathrm{Na}}$ [21]. As reported previously $[9,10,12,26]$, membrane ruptures created by MEP have large pores allowing permeation of large molecules and, therefore, are not selective for any particular organic and inorganic ions. To what extent pulsed electrical field-induced generation of noninactivating leak currents [30] is related to the formation of $I_{\text {MEP }}$ observed here remains to be further elucidated.

Previous reports have demonstrated the ability of PALCAR to increase the amplitude of late $\mathrm{Na}^{+}$currents and to decrease peak $\mathrm{Na}^{+}$currents in heart cells [31]. However, in our study, neither tetrodotoxin, ranolazine nor riluzole was demonstrated to have any effects on PALCAR-stimulated $I_{\text {MEP }}$ in $\mathrm{GH}_{3}$ cells. Those compounds are recognized to suppress the amplitude of late $\mathrm{Na}^{+}$currents $[32,33]$. PALCAR produced a progressive increase of $I_{\text {MEP }}$ in $\mathrm{GH}_{3}$ cells with an $\mathrm{EC}_{50}$ value of $2.4 \mu \mathrm{M}$. It thus seems unlikely that the PALCAR-mediated stimulation of $I_{\text {MEP }}$ presented here is primarily associated with its excitatory effects on the amplitude of late $\mathrm{Na}^{+}$current. Although PALCAR is reported to increase late $\mathrm{Na}^{+}$current [21], to what extent its stimulation of $I_{\mathrm{MEP}}$ in heart cells contributes to the generation of cardiac arrhythmias $[6,31]$ remains to be further investigated.

As an amphililic compound, PALCAR possesses a polar head-group that contains regions of positive and negative charge that are in close spatial proximity in certain molecular conformation [16]. When cells are exposed to PALCAR, it might readily incorporate into the phospholipid bilayer of the surface membrane. The PALCAR molecule contains a single aliphatic hydrocarbon chain which can be esterified to the polar head-group of the membrane. There are several indications showing that the pores of relatively large size induced by MEP are hydrophilic and that their inner surface is covered with polar heads of phospholipids. Additionally, by use of PCA in $I_{\mathrm{MEP}}$ fluctuations described here, it was found that the presence of PALCAR resulted in a drop without the appearance of heralded $I_{\min }$. Therefore, the incorporation of PALCAR into the membrane phospholipid bilayer may result in a significant perturbation of the orderly packing of adjacent phospholipid molecules which influence the $I_{\mathrm{MEP}}$ formation, resealing, or both. As the electrical field perturbs membrane lipid, electropores lined by the negatively charged head groups of phospholipids would be readily formed during cell exposure to long-chain acyl carnitines (e.g., PALCAR). The ability of PALCAR treatment to reduce the appearance of herald $I_{\min }$ observed in this study also lead us to propose that changes in the compositions of membrane phospholipid bilayers may alter the biophysical properties of MEP-associated currents in these cells. It also remains to be further investigated whether endogenous PALCAR or lysophosphatidylcholine [15, 34] can be a substrate used to enhance MEP-elicited channels which indirectly activate the intrinsic pathway to apoptotic or necrotic changes by inducing changes in the mitochondrial permeability transition pores $[2,3,35,36]$.

A recent report showed the suitability of patch-clamp experiments to assess $I_{\text {MEP }}$ in plant cells [37]. The magnitude of $I_{\text {MEP }}$ can control $\mathrm{Na}^{+}$or $\mathrm{Ca}^{2+}$ influx to increase with membrane hyperpolarization and fall with membrane depolarization owing to changes in electrochemical driving force. Activation of these channels can thus induce the firing of action potentials in excitable cells, or it regeneratively couples in a positive feedback relationship with $\mathrm{Na}^{+}$- or $\mathrm{Ca}^{2+}$-activated $\mathrm{K}^{+}$currents $[6,7,26,38]$. PALCAR has been previously reported to elevate the level of intracellular $\mathrm{Na}^{+}$or $\mathrm{Ca}^{2+}[20,31]$. Therefore, taken together, our study indicates that the increased activity of MEP-induced channels caused by PALCAR could be an important element in the modulation of functional activities of cells.

\section{Conflict of Interest}

The authors declare that they have no competing interests which are directly relevant to this study. 
So et al.: Analysis of Electroporation-Induced Currents

\section{Acknowledgements}

The authors wish to acknowledge Miss Wei-Hsin Yang for cell preparations in this work and are grateful to Dr. Eugenio Ragazzi (Department of Pharmacology, University of Padova, Italy) for his kind assistance in data analysis. They also thank the National Science Council of Taiwan for financial support of this work under contracts (NSC-101-2320-B-006-009 and NSC-101-2815-C-006-082-B).

\section{References}

1 Krassen H, Pliquett U, Neumann E: Nonlinear current-voltage relationship of the plasma membrane of single CHO cells. Bioelectrochemistry 2007;70:71-77.

- Vernier PT, Levine ZA, Wu YH, Joubert V, Ziegler MJ, Mir LM, Tieleman DP: Electroporating Fields Target Oxidatively Damaged Areas in the Cell Membrane. PLoS ONE 2009;4:e7966.

3 Sundararajan R: Nanosecond electroporation: another look. Mol Biotechnol 2009;41:69-82.

4 Wang M, Orwar O, Olofsson J, Weber SG: Single-cell electroporation. Anal Bioanal Chem 2010:397:32353248.

-5 Yu M, Tan W, Lin H: A stochastic model for DNA translocation through an electropore. Biochim Biophys Acta 2012;1818:2494-2501.

6 Akuzawa-Tateyama M, Tateyama M, Ochi R: Low K+-induced hyperpolarizations trigger transient depolarizations and action potentials in rabbit ventricular myocytes. J Physiol 1998;513:775-786.

7 Kaminska I, Kotulska M, Stecka A, Saczko J, Drag-Zalesinska M, Wysocka T, Choromanska A, Skolucka N, Nowicki R, Marczak J, Kulbacka J: Electroporation-induced changes in normal immature rat myoblasts (H9C2). Gen Physiol Biophys 2012;31:19-25.

-8 Otsuka H, Okimura S, Nagamura M, Matsukuma D, Kutsuzawa K, Matsuda N, Nakashima T, Okabe H: Effects of low-voltage pulse on cell elimination. Chem Lett 2012;41:1636-1638.

$>9$ Wu SN, Huang HC, Yeh CC, Yang WH, Lo YC: Inhibitory effect of memantine, an NMDA-receptor antagonist, on electroporation-induced inward currents in pituitary GH3 cells. Biochem Biophys Res Commun 2011;405:508-513.

10 Liu YC, Wu PC, Shieh DB, Wu SN: The effects of magnetite (Fe304) nanoparticles on electroporationinduced inward currents in pituitary tumor (GH3) cells and in RAW 264.7 macrophages. Int J Nanomedicine 2012;7:1687-1696.

11 Wu SN, Yeh CC, Wu PY, Huang HC, Tsai ML: Investigations into the correlation properties of membrane electroporation-induced inward currents: prediction of pore formation. Cell Biochem Biophys 2012;62:211-220.

12 Huang MH, Lin KH, Chen SJ, Shen AY, Wu FT, Wu SN: Effects of ketamine and its metabolites on ion currents in differentiated hippocampal H19-7 neuronal cells and in HEK293T cells transfected with $\alpha$-hslo subunit. Neurotoxicology 2012;33:1058-1066.

13 Ragazzi E, Pucciarelli S, Seraglia R, Molin L, Agostini M, Lise M, Traldi P, Nitti D: Multivariate analysis approach to the plasma protein profile of patients with advanced colorectal cancer. J Mass Spectrom 2006;41:1546-1553.

14 Lin JW: Latitude-time total electron content anomalies as precursors to Japan's large earthquakes associated with principal component analysis. Int J Geophysics 2011;2011:763527.

15 Su X, Han X, Mancuso DJ, Abendschein DR, Gross RW: Accumulation of long-chain acylcarnitine and 3-hydroxy acylcarnitine molecular species in diabetic myocardium: identification of alterations in mitochondrial fatty acid possessing in diabetic myocardium by shotgun lipidomics. Biochemistry 2005;44:5234-5245.

16 Mészáros J, Villanova L, Pappano AJ: Calcium ions and 1-palmitoyl carnitine reduce erythrocyte electrophoretic mobility: test of a surface charge hypothesis. J Mol Cell Cardiol 1988;20:481-492.

17 Tomita M, Doi N, Hayashi M: Effects of acylcarnitines on efflux transporting system in Caco-2 cell monolayers. Eur J Drug Metab Pharmacokinet 2010;35:1-7. 


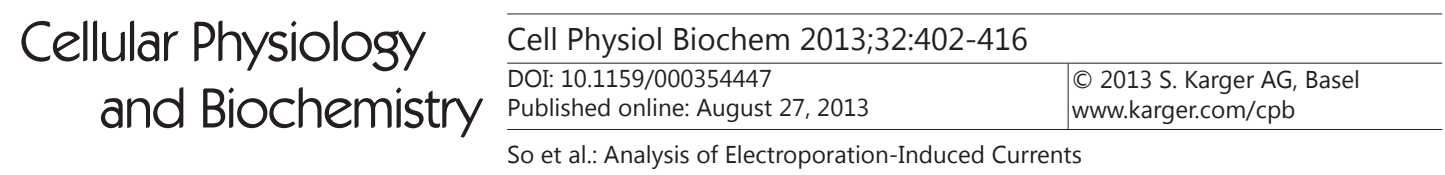

18 Netticadan T, Yu L, Dhalla NS, Panagia V: Palmitoyl carnitine increases intracellular calcium in adult rat cardiomyocytes. J Mol Cell Cardiol 1999;31:1357-1367.

19 Taki H, Muraki K, Imaizumi Y, Watanabe M: Mechanisms of the palmitoylcarnitine-induced response in vascular endothelial cells. Pflügers Arch 1999;438:463-469.

20 Muraki K, Imaizumi Y: A novel action of palmitoyl-L-carnitine in human vascular endothelial cells. J Pharmacol Sci 2003;92:252-258.

21 Wu Y, Song Y, Belardinelli L, Shryock JC: The late $\mathrm{Na}^{+}$current (INa) inhibitor ranolazine attenuates effects of palmitoyl-L-carnitine to increase late INa and cause ventricular diastolic dysfunction. J Pharmacol Exp Ther 2009;330:550-557.

-22 Sabirov RZ, Merzlyak PK: Plasmalemmal VDAC controversies and maxi-anion channel puzzle. Biochim Biophys Acta 2012;1818:1570-1580.

23 Wu SN, Wu PY, Tsai ML: Characterization of TRPM8-like channels activated by the cooling agent icilin in the macrophage cell line RAW 264.7. J Membr Biol 2011;241:11-20.

24 Wu SN, Yu HS, Jan CR, Li HF, Yu CL: Inhibitory effects of berberine on voltage- and calcium-activated potassium currents in human myeloma cells. Life Sci 1998;62:2283-2294.

25 Kemmer G, Keller S: Nonlinear least-squares data fitting in Excel spreadsheets. Nat Protocol 2010;5:267281.

26 Dyachok 0, Zhabyeyev P, McDonald TF: Electroporation-induced inward current in voltage-clamped guinea pig ventricular myocytes. J Membr Biol 2010;238:69-80.

27 Kinoshita H, Matsuda N, Iranami H, Ogawa K, Hatakeyama N, Azma T, Kawahito S, Yamazaki M: Isoflurane pretreatment preserves adenosine triphosphate-sensitive $\mathrm{K}^{+}$channel function in the human artery exposed to oxidative stress caused by high glucose levels. Anesth Analg 2012;115:54-61.

-28 Nesin V, Pakhomov AG: Inhibition of voltage-gated $\mathrm{Na}^{+}$current by nanosecond pulsed electric field (nsPEF) is not mediated by $\mathrm{Na}^{+}$influx or $\mathrm{Ca}^{2+}$ signaling. Bioelectromagnetics 2012;33:443-451.

29 Verkerk AO, van Ginnekern ACG, Wilders R: Sodium current inhibition by nanosecond pulsed electrical field (nsPEF) - fact or artifact? Bioelectromagnetics 2013;34:162-164.

30 Nesin V, Bowman AM, Xiao S, Pakhomov AG: Cell permeabilization and inhibition of voltage-gated $\mathrm{Ca}^{2+}$ and $\mathrm{Na}+$ channel currents by nanosecond pulsed electric field. Bioelectromagnetics 2012;33:394-404.

31 Wu J, Corr PB: Palmitoylcarnitine increases $\left[\mathrm{Na}^{+}\right]_{\mathrm{i}}$ and initiates transient inward current in adult ventricular myocytes. Am J Physiol Heart Circ Physiol 1995;268:H2405-2417.

32 Chen BS, Lo YC, Peng H, Hsu TI, Wu SN: Effects of ranolazine, a novel anti-anginal drug, on ion currents and membrane potential in pituitary tumor GH3 cells and NG108-15 neuronal cells. J Pharmacol Sci 2009;110:295-305.

-33 Wu SN, Yeh CC, Huang HC, So EC, Lo YC: Electrophysiological characterization of sodium-activated potassium channels in NG108-15 and NSC-34 motor neuron-like cells. Acta Physiol 2012;206:120-134.

-34 Tominaga H, Katoh H, Odagiri K, Takeuchi Y, Kawashima H, Saotome M, Urushida T, Satoh H, Hayashi H: Different effects of palmitoyl-L-carnitine and palmitoyl-CoA on mitochondrial function in rat ventricular myocytes. Am J Physiol Heart Circ Physiol 2008;295:H105-112.

-35 Del Principe D, Avigliano L, Savini I, Catani MV: Trans-plasma membrane electron transport in mammals: functional significance in health and disease. Antioxid Redox Signal 2011;14:2289-2318.

-36 Rinaldelli E, Panattoni A, Luvisi A, Triolo E: Effect of mycophenolic acid on trans-plasma membrane electron transport and electric potential in virus-infected plant tissue. Plant Physiol Biochem 2012;60:137140 .

37 Wegner LH, Frey W, Schönwälder S: A critical evaluation of whole cell patch clamp studies on electroporation using the voltage sensitive dye ANNINE-6. Bioelectrochemistry 2013;92:42-46.

38 Lou Q, Fedorov VV: Shock-induced focal arrhythmias: Not driven by calcium? Heart Rhythm 2012;9:105106. 